determine immediately that the spin-orbit splitting is $61.5 \pm 1.0 \mathrm{~cm}^{-1}$. Further analysis of the rotational structure of these bands may be expected to refine this, but should not change it by more than two wave numbers.

In addition, a preliminary analysis of the vibrational progressions provides strong evidence that the most intense, highest frequency spin-orbit component of the origin band is actually at $32306 \pm 2 \mathrm{~cm}^{-2}$, rather than in the region $31540 \mathrm{~cm}^{-1}$ supposed by other workers. This fact could have easily been missed in previous work because both of the origin components adjoin strong $\mathrm{OH}$ lines!

The value of the spin-orbit splitting observed is closer to the value determined from the LMR data ${ }^{9}$ rather than to that of the theoretical prediction. ${ }^{13}$ This theory should be reexamined. Of the three possible theoretical influences on the spin-orbit splitting, we suspect that the orbital quenching in this radical is not as large as supposed. Two pieces of information point to this. First, preliminary anlysis of the spectra is consistent with a total Jahn-Teller coupling of about $k^{2}=0.5$, which could account for almost all of the reduction from about 140 to $60 \mathrm{~cm}^{-1}$. Second, orbital quenching would require significant delocalization of the valence hole on oxygen onto the hydrogens. The fact that the transition origin is almost exactly at the energy of the analogous transition in $\mathrm{OH}$ suggests that the transition is entirely located on the oxygen, with little shift caused by hyperconjugation onto the hydrogens.

We have not yet completed an analysis of the methoxy emission system. A full vibronic analysis of the spectrum and that of the deuterated species should provide an understanding of the Jahn-Teller effect and the mechanism of orbital quenching or reduction in this simple system.
We thank the Murdock Foundation for support of this project.

${ }^{1}$ T. Ebata, H. Yanagishita, K. Obi, and I. Tanaka, Chem. Phys. 69, 27 (1982).

${ }^{2}$ P. C. Engelking, G. B. Ellison, and W. C. Lineberger, J. Chem. Phys. 69, 1826 (1978).

${ }^{3}$ G. Inoue, H. Akimoto, and M. Okuda, Chem. Phys. Lett. 63, 213 (1979).

${ }^{4}$ G. Inoue, H. Akimoto, and M. Okuda, J. Chem. Phys. 72, 1769 (1980).

${ }^{5}$ M. Iwasaki and K. Toriyama, J. Am. Chem. Soc. 100, 1964 (1978).

${ }^{6} \mathrm{~K}$. Ohbayashi, H. Akimoto, and I. Tanaka, J. Phys. Chem. 81, 798 (1977).

${ }^{7}$ D. E. Powers, J. B. Hopkins, and R. E. Smalley, J. Phys. Chem. 85, 2711 (1981).

${ }^{8}$ H. E. Radford and D. K. Russell, J. Chem. Phys. 66, 2222 (1977).

${ }^{9}$ D. K. Russell and H. E. Radford, J. Chem. Phys. 72, 2750 (1980).

${ }^{10}$ M. Sutoh, N. Washida, H. Akimoto, M. Nakamura, and M. Okuda, J. Chem. Phys. 73, 591 (1980).

${ }^{11}$ H. R. Wendt and H. E. Hunziker, J. Chem. Phys. 71, 5202 (1979).

${ }^{12}$ B. K. Janousek, A. H. Zimmerman, K. J. Reed, and J. I. Brauman, J. Am. Chem. Soc. 100, 642 (1978).

${ }^{13}$ G. D. Bent, G. F. Adams, R. H. Bartram, G. D. Purvis, and R. J. Bartlett,

J. Chem. Phys. 76, 4144 (1982).

${ }^{14}$ J. K. Watson, J. Mol. Spectrosc. 103, 125 (1984).

${ }^{15}$ J. M. Brown, Mol. Phys. 20, 817 (1971).

${ }^{16} \mathrm{R}$. Englman, The Jahn-Teller Effect in Molecules and Crystals (Wiley, New York, 1972).

${ }^{17}$ S. M. Colwell, Mol. Phys. 51, 1217 (1984).

${ }^{18}$ S. M. Colwell, R. D. Amos, and N. C. Handy, Chem. Phys. Lett. 109, 525 (1984)

${ }^{19}$ D. R. Yarkony, H. F. Schaefer III, and S. Rothenberg, J. Am. Chem. Soc. 96, $656(1974)$.

${ }^{20}$ P. G. Carrick and P. C. Engelking, J. Chem. Phys. 81, 1661 (1984).

${ }^{2}$ P. G. Carrick and P. C. Engelking, Chem. Phys. Lett. 108, $505(1984)$

${ }^{22}$ A. T. Droege and P. C. Engelking, Chem. Phys. Lett. 96, 316 (1983).

${ }^{23}$ P. L. Hanst and J. C. Calvert, J. Phys. Chem. 63, $104(1959)$.

\title{
Picosecond monitoring of a chemical reaction in molecular beams: Photofragmentation of $\mathbf{R}-\mathbf{I} \rightarrow \mathbf{R}^{\ddagger}+\mathbf{I}$
}

\author{
J. L. Knee, L. R. Khundkar, and A. H. Zewaila)

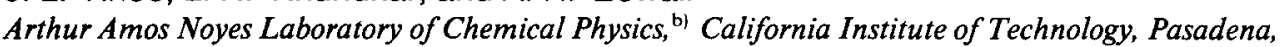 \\ California 91125
}

(Received 15 May 1985; accepted 11 June 1985)

Studies of chemical reactions by molecular beam techniques and spectroscopic methods have provided extensive information on the nature of the intermediates involved and on the energy distribution in the products. ${ }^{1-6}$ Until now, however, there is no report of direct picosecond time resolution of a chemical reaction in molecular beams.

In this communication, we report our first picosecond experimental results on photofragmentation reactions with product state detection. The experiments are performed using the pump-probe picosecond/mass-spectrometry tech-

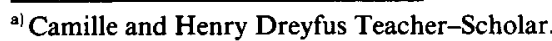

${ }^{b)}$ Contribution No. 7189.
}

nique. ${ }^{7}$ Two picosecond pulses, delayed in time by a Michelson interferometer, are spatially overlapped in a pulsed molecular beam; the pump pulse initiates the photofragmentation while the probe pulse monitors the dynamics of product state formation using TOF mass spectrometry (see Fig. 1). The reactions studied are those of alkyl iodides, which have been extensively studied by photofragment spectroscopy $^{1,8}$ :

$$
\mathrm{R}-\mathrm{I} \rightarrow \mathrm{R}^{\ddagger}+\mathrm{I}\left({ }^{2} P_{3 / 2} \text { or }{ }^{2} P_{1 / 2}\right) .
$$

In this report $\mathrm{R}$ stands for $\mathrm{CH}_{3}$ or $\mathrm{CF}_{2} \mathrm{I}-\mathrm{CF}_{2}$. The two spinorbit states of the iodine atom are probed separately by tun- 
ing the wavelength of the probe pulse (two-photon absorption) to $304.0\left({ }^{2} P_{1 / 2}\right)$ or $304.7 \mathrm{~nm}\left({ }^{2} P_{3 / 2}\right)$. The results show that for $\mathrm{CH}_{3} \mathrm{I}$ the primary $\mathrm{C}-\mathrm{I}$ bond breakage is occurring within our pulse duration, while for $\mathrm{CF}_{2} \mathrm{I}-\mathrm{CF}_{2} \mathrm{I}$ there is a sequential bond breakage; a similar primary and a slower (32 ps) secondary C-I breakage. The scheme for our experiments is illustrated in Fig. 1.

Methyl iodide, whose C-I $n \rightarrow \sigma^{*}$ transition is at $\sim 2800$ $\AA$, is known to photofragment and form iodine in both the $1 / 2$ and $3 / 2$ states. ${ }^{1}$ The $\mathrm{CH}_{3}$ fragment produced is vibrationally excited. ${ }^{9}$ Figure 2 shows results obtained by probing $I *\left({ }^{2} P_{1 / 2}\right)$ and similar results (not shown) were obtained when probing $I\left({ }^{2} P_{3 / 2}\right)$. In both cases, the iodine signal rises within the pulse and we can fit our data to a buildup time of $0.5 \mathrm{ps}$. The photodissociation lifetime has been estimated ${ }^{8}$ to be on the order of a rotational period, and our results for $\mathrm{CH}_{3} \mathrm{I}$ indicate that $\tau \leqslant 0.5 \mathrm{ps}$.

In Fig. 2, we also show the results for $\mathrm{I}-\mathrm{CF}_{2}-\mathrm{CF}_{2}-\mathrm{I}{ }^{10}$ When $I^{*}$ was monitored the signal rose within the pulse similar to the observation made on $\mathrm{CH}_{3} \mathrm{I}$. On the other hand, when I was monitored a "biexponential" buildup was observed; the least-squares fit gives $\tau_{1}=0.5$ and $\tau_{2}=32 \pm 10$ ps.

The above preliminary results indicate the following: First, the primary $\mathrm{C}-\mathrm{I}$ bond breakage in $\mathrm{CH}_{3} \mathrm{I}$ occurs within the time resolution of our picosecond/mass spectrometry

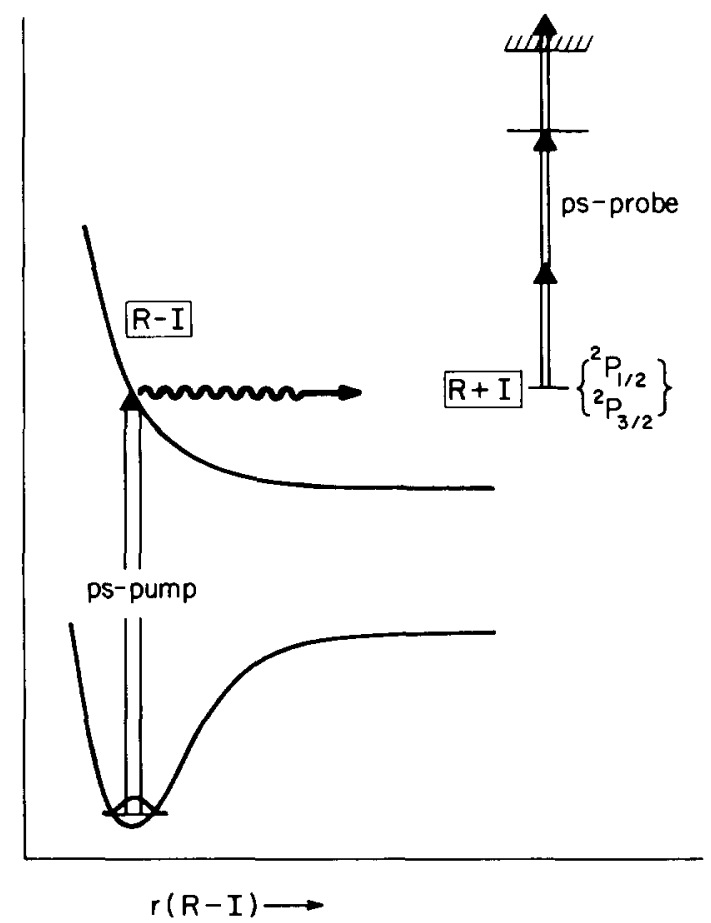

FIG. 1. Generalized energy level diagram describing the ps pump-probe experiment on the photofragmentation of R-I. The pump pulse (frequency doubled dye laser at $2800 \AA$ ) excites the molecules to the repulsive surface of the $\mathrm{C}-\mathrm{I}$. The probe (generated by Raman shifting in methane) monitors the population of iodine in the $1 / 2$ state $(304.0 \mathrm{~nm})$ or the $3 / 2$ state $(304.7 \mathrm{~nm})$ using TOF mass spectrometry. Details of I atom transitions, detected by MPI, are given in Ref. 19.

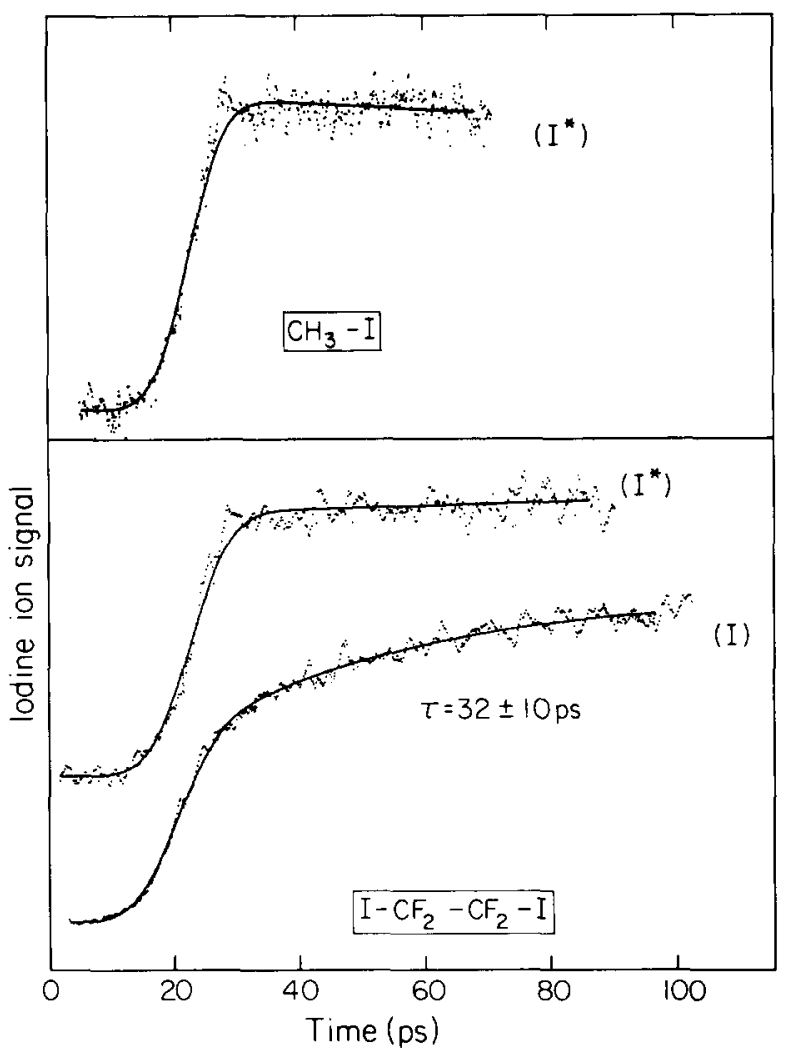

FIG. 2. Picosecond transient detection of product $I$ or I* in the photofragmentation of a beam of $\mathrm{CH}_{3} \mathrm{I}$ or $\mathrm{CF}_{2} \mathrm{I}-\mathrm{CF}_{2} \mathrm{I}$. The vertical scale represents the iodine ion signal while the horizontal one gives the delay time between the pump and probe ps pulses. The beam conditions are: stagnation pressure $\simeq 20$ psi of helium; sample temperature is maintained at $0^{\circ} \mathrm{C}$. The laser pulses cross the molecular beam $10 \mathrm{~cm}$ downstream from the pulsed nozzle and $9 \mathrm{~cm}$ from the skimmer. Approximately $10^{3} \mathrm{I}$ atoms are detected per pulse. Top trace: transients for $\mathrm{CH}_{3} \mathrm{I}$ obtained by detecting I* (similar transient was obtained when monitoring I). Bottom traces: transients for $\mathrm{CF}_{2} \mathbf{I}-$ $\mathrm{CF}_{2} \mathrm{I}$ obtained by monitoring I or $\mathrm{I}^{*}$. The solid lines are the least-squares fit of the data (see text).

when either I or $I^{*}$ is monitored. Second, in $I-C F_{2}-C F_{2}-I$, the buildup time is $32 \mathrm{ps}$ for ground state iodine and very short for the excited iodine. These observations can be understood in terms of a simple sequential bond breakage: a prompt photofragmentation on the repulsive surface (Fig. 1) occurs first, ${ }^{11}$ followed by a secondary bond breakage of the other C-I bond. The dynamics of this prompt breakage can be understood using available theoretical techniques. ${ }^{14}$ The wave packet approach, ${ }^{15}$ as highlighted recently by the work at MIT, ${ }^{16}$ indicates that the lifetime is less than a picosecond. The question now: what determines the dynamics of this slower secondary process?

The observation of a $30 \mathrm{ps}$ rise by monitoring I (and not $\left.I^{*}\right)$ indicates that after the recoil of the fragments in the primary fragmentation the total internal energy in $R^{\ddagger}$ is sufficient for the radical to undergo secondary dissociation and produce I in the ground state. Lee's group ${ }^{3}$ has done a thorough study of the translational energy distribution for similar reactions. From the photon energy $(102 \mathrm{kcal} / \mathrm{mol})$ of our experiment, the $\mathrm{C}-\mathrm{I}$ bond energy $(52.5 \mathrm{kcal} / \mathrm{mol})$ and the mean translational energy ${ }^{3}$ of similar systems we conclude that the internal energy of the fragment is comparable to the activation energy and that bond breakage of the second io- 
dine takes place. The $(30 \mathrm{ps})^{-1}$ represents the average rate for this secondary bond breaking process. This is consistent with the fact that this buildup was only seen when I (and not I*) was monitored.

The observed "biexponential" buildup of I (Fig. 2) can be related to the internal energy distribution in $\mathrm{CF}_{2} \mathrm{I}-\mathrm{CF}_{2}$. Assuming a thermal vibrational distribution (width of 6 $\mathrm{kcal} / \mathrm{mol})^{3}$ that peaks near the barrier (taken from thermochemical estimates to be $3-5 \mathrm{kcal} / \mathrm{mol}$ ), and taking the dependence of the rates on excess energy $\left(E_{x}\right)$ to be of the form $A e^{-\alpha / E_{x}{ }^{17}}$ we obtain a biexponential buildup from this convolution procedure, consistent with our experimental findings. We are currently examining details of the fragmentation pathways, possible surface crossing, and RRKM calculations.

In conclusion, picosecond probing of reactions in beams offers new opportunities for real-time studies of the dynamics of bond breakage. This first report confirms our optimism about the sensitivity of the method which provides excellent time resolution and allows for product state analysis since two color picosecond pulses are used. Extension to other systems, including those studied by Parker and Bernstein, ${ }^{18}$ is currently in progress. We are also extending these studies to the femtosecond time domain.

This research was supported by a grant from the National Science Foundation. We thank Professor R. Marcus, Professor R. Bernstein, Professor W. Goddard, and Professor J. Beauchamp for enlightening discussions. This work was presented at the International Conference "Recent Advances in Molecular Reaction Dynamics," Aussois, France, June 1985.

${ }^{1}$ For more reviews, see Refs. $2-5$, and the book by R. B. Bernstein, Chemical Dynamics via Molecular Beam and Laser Techniques (Clarendon/Oxford University, Oxford, 1982).
${ }^{2}$ Y. T. Lee, Laser Chem. 2, 219 (1983); R. N. Zare and R. B. Bernstein, Phys. Today, p. 43, 1980.

${ }^{3}$ D. Krajnovich, L. T. Butler, and Y. T. Lee, J. Chem. Phys. 81, 3031 (1984), and references therein.

${ }^{4}$ R. Bersohn, J. Phys. Chem. 88, 5145 (1984).

${ }^{5} J$. P. Simons, J. Phys. Chem. 88, 1287 (1984).

${ }^{6}$ H. J. Foth, H. R. Mayne, R. A. Poirier, J. C. Polanyi, and H. H. Telle, Laser Chem. 2, 229 (1983).

${ }^{7}$ J. L. Knee, F. E. Doany, and A. H. Zewail, J. Chem. Phys. 82, 1041 (1985); J. L. Knee, L. R. Khundkar, and A. H. Zewail, J. Chem. Phys. 82, 4715 (1985).

${ }^{8}$ (a) S. J. Riley and K. R. Wilson, Faraday Discuss. Chem. Soc. 53, 132 (1972); (b) M. Dzronik, S. Yang, and R. Bersohn, J. Chem. Phys. 61, 4408 (1974).

${ }^{9}$ H. W. Hermann and S. R. Leone, J. Chem. Phys. 76, 4759, 4766(1982); see also J. Chem. Phys. 72, 6531 (1980).

${ }^{10}$ The absorption spectrum at room temperature shows a broad band at 265 nm. This compound, obtained from PCR Specialty Chemicals, was used without further purification. The GC, UV absorption, and mass spectrum are all consistent with a purity (other than $I_{2}$ ) of $98 \%$ or better. $I_{2}$, however, absorbs weakly at $\lambda$ of interest and has a much lower vapor pressure [Ref. 8(a)].

"In these experiments, the observed fragment is $\mathrm{I}^{+}$and there is no detectable $\mathrm{I}_{2}^{+}$, indicating that there is no concerted bond breakage. This is consistent with results on di-iodomethane by Kawasaki et al. (Ref. 12) and Kroger et al. (Ref. 13).

${ }^{12}$ M. Kawasaki, S. J. Lee, and R. Bersohn, J. Chem. Phys. 63, 809 (1975). These authors presented a clear discussion of the reasons for consecutive bond breakage in di-iodomethane, where resonance interactions between the two $\mathrm{C}-\mathrm{I}$ bonds is quite strong.

${ }^{13}$ P. M. Kroger, P. C. Demou, and S. J. Riley, J. Chem. Phys. 65, 1823 (1976).

${ }^{14}$ For reviews, see M. Shapiro and R. Bersohn, J. Chem. Phys. 73, 3810 (1980); K. F. Freed and Y. B. Band, Excited States 3, 109 (1978); W. M. Gelbart, Annu. Rev. Phys. Chem. 28, 323 (1977).

${ }^{15}$ E. J. Heller, Acc. Chem. Res. 14, 368 (1981); S. Y. Lee and E. J. Heller, J. Chem. Phys. 76, 3035 (1982).

${ }^{16}$ D. Imre, J. L. Kinsey, A. Sinha, and K. Krenos, J. Phys. Chem. 88, 3956 (1984).

${ }^{17}$ A. H. Zewail, Faraday Discuss. Chem. Soc. 75, 315 (1983).

${ }^{18}$ D. H. Parker and R. B. Bernstein, J. Phys. Chem. 86, 60 (1982).

${ }^{19}$ A. Gedanken, M. B. Robin, and Y. Yafet, J. Chem. Phys. 76, 4798 (1982); see also C. E. Moore, Natl. Bur. Stand. (U.S.) Circ. 35 (1971).

\title{
NOTES
}

\section{Direct measurement of the stop gap for the $31475 \mathrm{~cm}^{-1}$ singlet a exciton of naphthalene ${ }^{a)}$}

\author{
Sylvia H. Stevenson ${ }^{\text {b) }}$ and Gerald J. Small \\ Ames Laboratory-USDOE and Department of Chemistry, Iowa State University, Ames, Iowa 50011
}

(Received 18 March 1985; accepted 29 April 1985)

Recently, ${ }^{1-4}$ we have utilized phase-matched two-photon excitation or polariton fusion ${ }^{5-8}$ to study the spatial dispersion and scattering properties of the polariton associated with the lowest singlet $a$ exciton of naphthalene at $\omega_{T}$ $=31475 \mathrm{~cm}^{-1}$. For reasons noted below, these nonlinear results stimulated the present study which deals with the one-photon fluorescence excitation profile from the $a$ exciton.

The $a$ exciton possesses $A_{\mu}$ factor group $\left(C_{2 h}\right)$ symmetry and an oscillator strength $F$ of only $2.3 \times 10^{-5}$ (Ref. 4) and lies at the bottom of the band. In the nonlinear experi- ments, ${ }^{1-4}$ both the SHG (second harmonic generation) and TPA (two-photon absorption) resonant profiles were monitored at a resolution of $\sim 0.06 \mathrm{~cm}^{-1}$. Both processes are weakly allowed via a magnetic dipole-electric dipole mechanism. ${ }^{9}$ Accurate determinations of the upper and lower polariton branches were made and the curves fit theoretically with $F$ fixed at the above value (determined from one-photon absorption measurements ${ }^{4}$ ). The analytic expressions for the dispersion curves were used to show that polaritons with group velocities between $\sim 5 \times 10^{5}$ and $\sim 5 \times 10^{8} \mathrm{~cm} \mathrm{~s}^{-1}$ can be generated by angle tuning of $a b$ plate crystals about the $b$ 